\title{
Case Study: Rapid Training of Community Health Workers Using Enskill ${ }^{\circledR}$
}

https://doi.org/10.3991/ijac.v14i2.25929

\author{
W. Lewis Johnson ${ }^{(凶)}$ \\ Alelo Inc., Los Angeles, CA, USA \\ ljohnsonealelo.com
}

\begin{abstract}
The COVID-19 pandemic caused many workers to lose their jobs and also resulted in rapid surges in demand for workers with particular skills. In public health, there was suddenly a huge demand for community health workers to conduct contact tracing, vaccination, and community outreach. To address this need, our team undertook the challenge of creating an online course that trains workers for community health work in half the time of typical training programs. It utilizes the Enskill ${ }^{\mathbb{B}}$ learning platform, which uses artificial intelligence (AI) technology to create simulated scenarios in which trainees practice skills with avatars acting as simulated patients. Fifty-seven training participants without college degrees were recruited for the program from the Hampton Roads region, in collaboration with the Hampton Roads Workforce Council. The first cohorts of trainees were able to complete the training successfully in just eight weeks, and they were then placed in public health and healthcare positions. The approach also shows promise for upskilling existing employees to address skill gaps. The Enskill training program is a competitor in the XPRIZE Foundation's Rapid Reskilling competition, to quickly reskill under-resourced workers for the digital revolution.
\end{abstract}

Keywords—upskilling, reskilling, artificial intelligence, avatars

\section{Introduction}

The COVID-19 pandemic caused huge dislocations in the economy. Many workers lost their jobs at the start of the pandemic, and a 2021 Pew Research Study found that two-thirds of unemployed individuals seriously considered switching occupations [3]. At the same time, there were sudden demands for people to fill new jobs. In June 2020, the United States had 28,000 contact tracers, but the Center for Disease Control estimated that 100,000 were needed [4]. As workers returned to work, new labor shortages emerged in a number of sectors, and many of them persist [6].

These problems have heightened awareness of the skill gaps in the global workforce and the need to reskill and upskill workers [1]. Reskilling/upskilling are the top concerns of learning and development professionals, according to the 2021 L\&D Global Sentiment Survey [5]. To address this challenge, the XPRIZE Rapid Reskilling (XPRR) competition [7] was established. This \$5 million, 30-month competition seeks 
solutions that quickly reskill disadvantaged workers for the digital revolution. Solutions should reskill workers in half the time of conventional training and prepare them for living-wage jobs.

As part of the XPRR competition, we developed an online course that quickly retrains workers to become community health workers. The course runs on the Enskill ${ }^{\circledR}$ artificial intelligence-driven (AI-driven) learning platform. The first trainees completed the course in just eight weeks and were then placed in jobs in the Hampton Roads region.

\section{Enskill and its application to community health work}

Enskill is a cloud-based learning platform that employs AI in multiple ways to facilitate rapid skill development. Trainees learn new skills by practicing them with AI-driven avatars in realistic simulations of situations in the workplace and in everyday life. Trainees engage in spoken conversations with the avatars, much as they would with people in real life. They do not follow a script but are free to express themselves in their own words. As the trainee interacts with the avatar, Enskill continually evaluates the trainee's performance and, at the conclusion of the conversation, provides feedback about what they did well and what they need to improve. Enskill then recommends practice exercises relating to the skills that the trainee needs to improve. Trainees can practice conversations as many times as necessary to develop full mastery of the target skills.

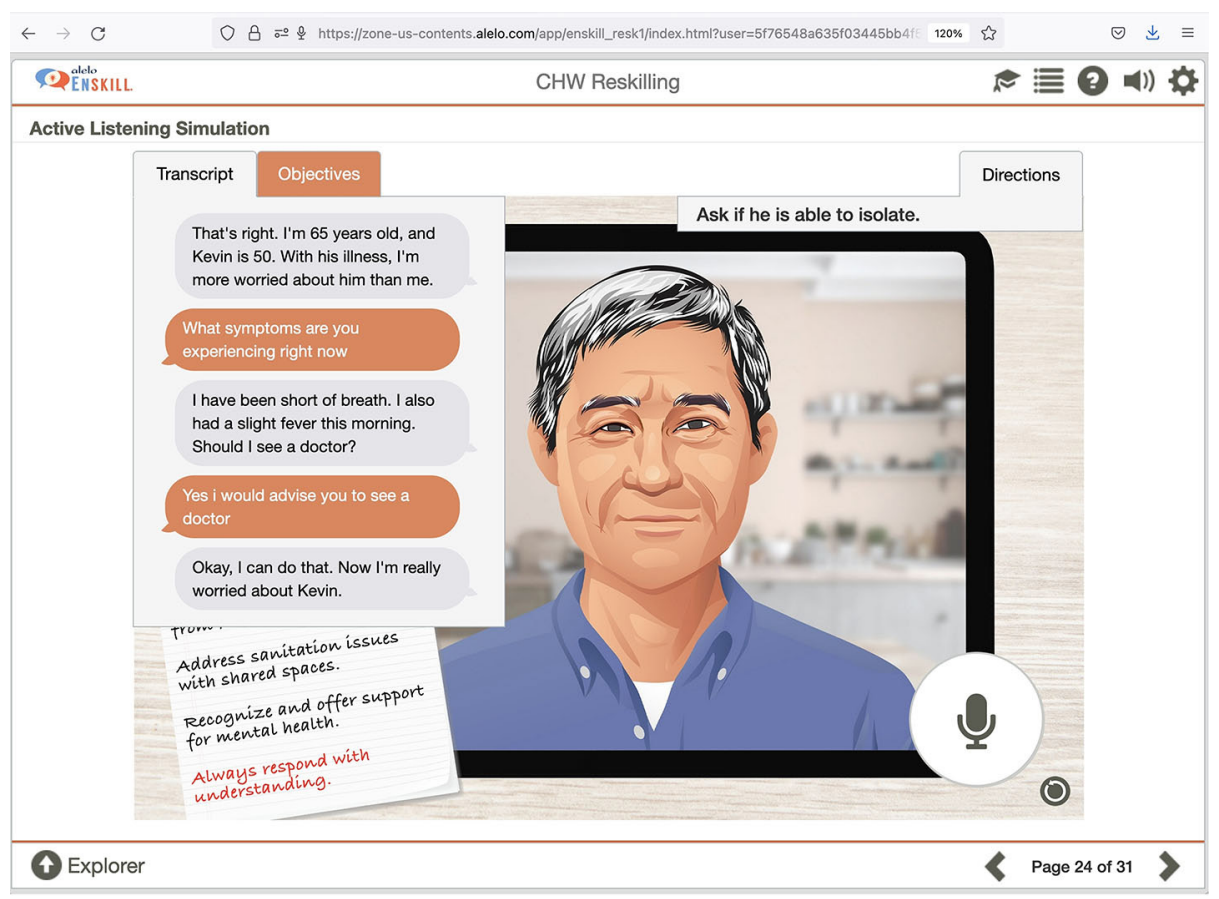

Fig. 1. Screenshot from the beginning of an Enskill practice conversation 
Practicing with Enskill conversations promotes learning in the following ways. Because learners must immediately apply what they learned in conversation, it immediately becomes apparent if a trainee skimmed through learning material and failed to retain it. For example, in some simulations, the trainee must explain to the avatar what they have just learned-e.g., the difference between quarantine and isolation. Practice in realistic simulations helps the trainees transfer the knowledge they have acquired to real-world settings. Practice in simulations also helps trainees build self-confidence, so when they encounter similar situations on the job, they feel that they know how to handle them.

Enskill was originally developed to help develop spoken proficiency in foreign languages [2]. We felt that it would be a good choice for training community health workers (CHWs) because effective communication is a key CHW competency. We hypothesized that trainees who practiced communication skills with our avatars would be better prepared to engage with real patients on the job.

Figures 1, 2, and 3 show screenshots from the CHW course. In Figure 1, the trainee engages in a follow-up video call with an avatar who previously had been exposed to COVID-19. The trainee is following up with the patient to find out whether the patient, Gary, is showing symptoms of COVID-19 and needs to isolate himself from other family members. The transcript of the conversation appears on the left side of the screen.

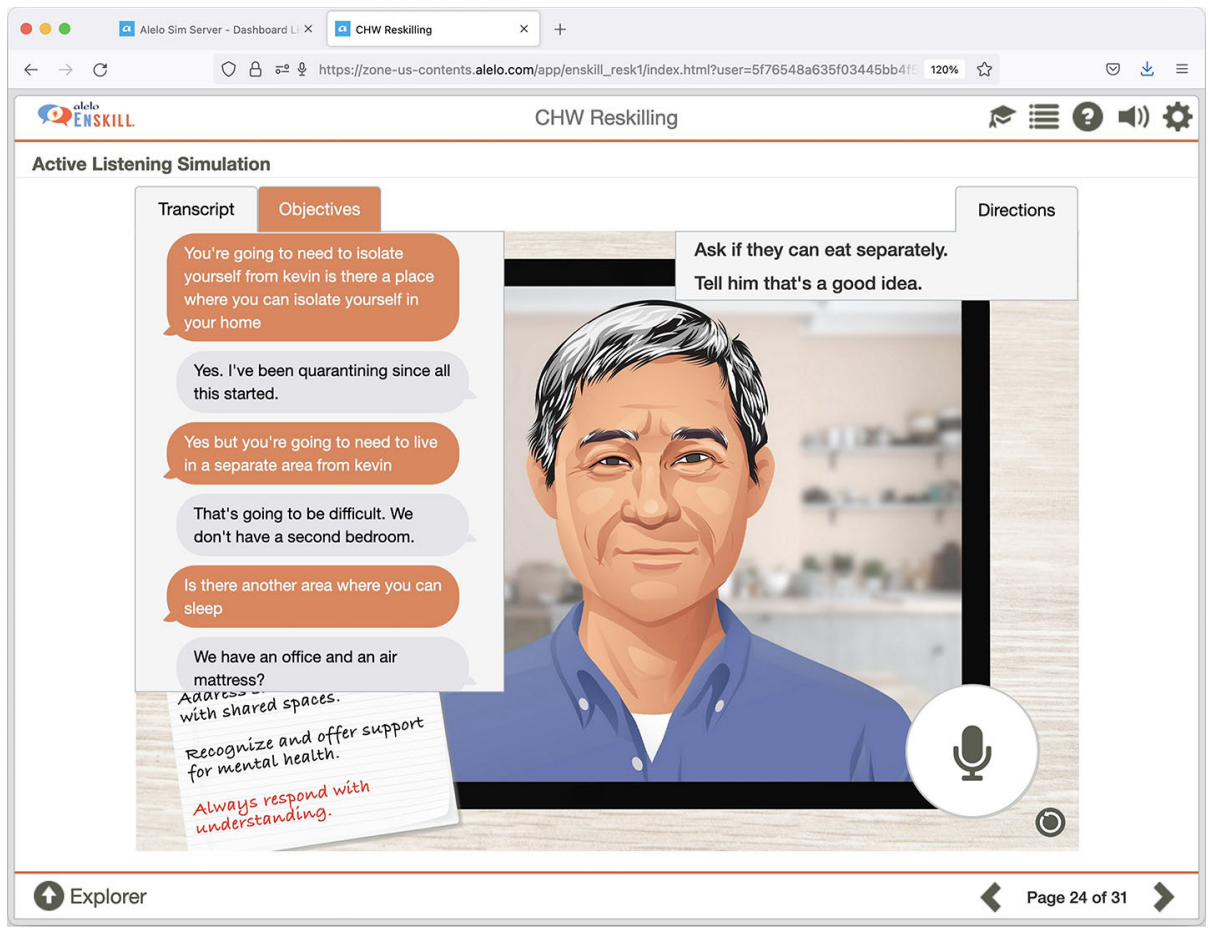

Fig. 2. Continuing the Enskill practice conversation 
Figure 2 shows a later point in the conversation. The trainee has informed Gary that he will need to isolate himself, and Gary responds that he has been quarantining. This suggests that Gary may not understand the difference between quarantining (i.e., staying home) and self-isolating (i.e., avoiding all contact with others, including family members). Therefore, the trainee explains that Gary will need to sleep separately and live in a separate part of the house from his husband. The trainee is, thus, demonstrating that she is able to respond to what she hears from the patient and is able to apply communication skills that are appropriate for the situation.

Figure 3 shows the feedback that the trainee receives at the end of the simulation. The trainee has successfully met all of the simulation objectives. If the trainee fails to meet the objectives, Enskill can recommend practice exercises focusing on the skills the trainee had difficulty with. We encourage trainees to keep practicing until they can successfully meet all of the objectives of each simulation.

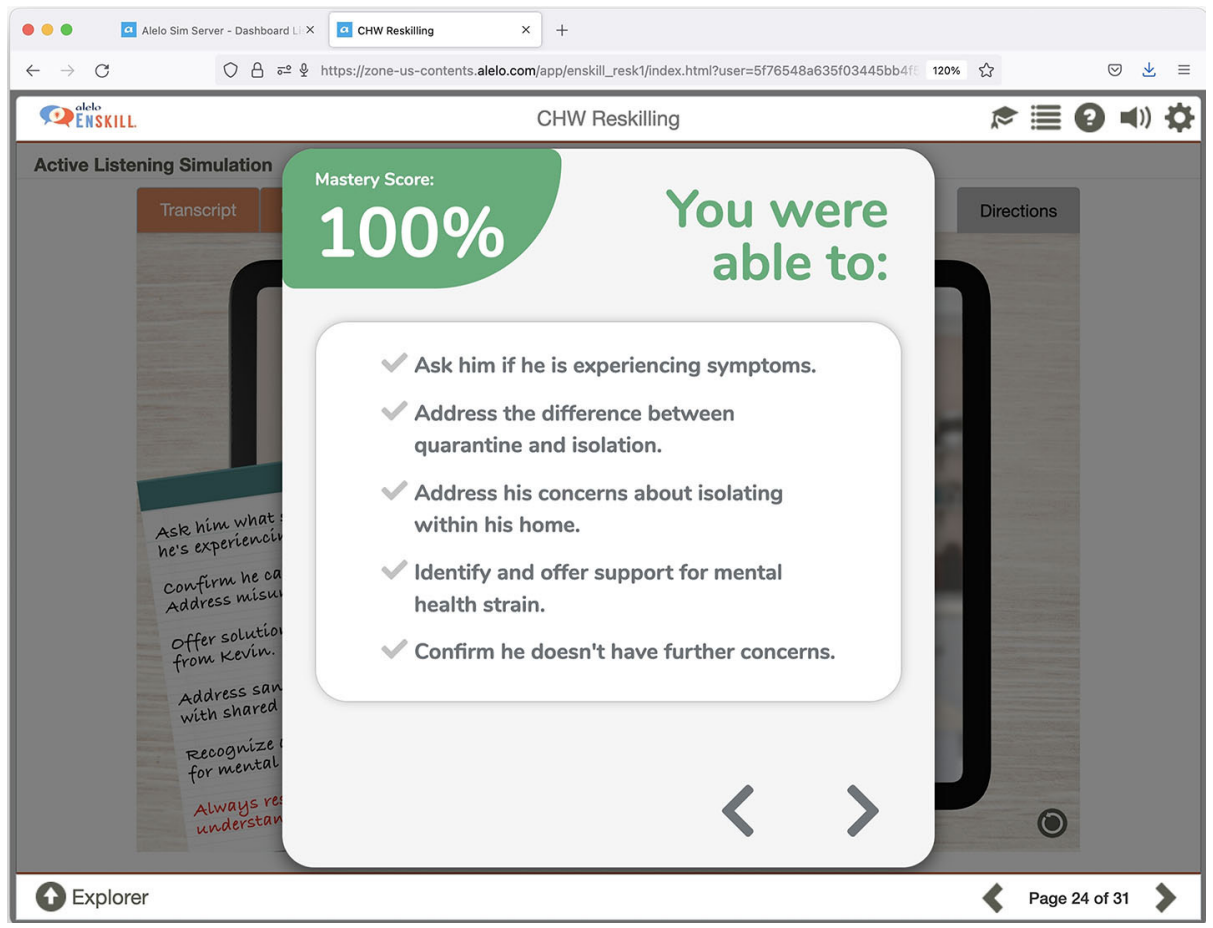

Fig. 3. Feedback for the trainee at the end of the simulation

The complete course combines self-paced training in Enskill with webinars that provide background information, as well as with peer exercises in which trainees can further practice their skills in role-plays with other trainees. Instructors observe trainees' performances to confirm that they have mastered the core competencies in the course. 


\section{Training results}

The training course was piloted with underemployed workers in the Hampton Roads region of Virginia, in collaboration with the Hampton Roads Workforce Council. A total of 57 trainees initiated the training. None of the trainees had completed a college degree. The trainees who completed the training in eight weeks or less, working part-time, had a $40 \%$ completion rate, which is as good or better than the typical online training program.

Both the avatar exercises and the peer role-play exercises were judged positively by the trainees. The combination of avatar and peer exercises proved to be a very effective approach. The avatar exercises provided trainees with an opportunity to develop their skills in a safe environment, without fear of embarrassing themselves. The peer exercises then helped trainees further improve their communication skills and demonstrate mastery.

We are now helping trainees to get jobs in public health and healthcare in the Hampton Roads region. We will follow up with the employers to make sure that the trainees are able to apply their skills successfully on the job. Assuming that the placement is successful, we anticipate advancing to the next round in the XPRIZE competition, in which we apply Enskill to additional occupations and industries. We plan to target sales and customer service occupations, which also require effective communication skills.

\section{Acknowledgment}

We wish to acknowledge the support of the XPRIZE Foundation. The prize money that we won in the XPRIZE Rapid Reskilling competition helped fund this project.

\section{$5 \quad$ References}

[1] Agarwal, S., De Smet, A., Poplawski, P., and Reich, A. (2020). Beyond hiring: How companies are reskilling to address talent gaps. McKinsey \& Company.

[2] Johnson, W.L. Data-driven development and evaluation of Enskill English. International Journal of AI in Education 29: 425-457. https://doi.org/10.1007/s40593-019-00182-2

[3] Parker, K., Igielnik, R. and Kochhar, R. (2021). Unemployed Americans are feeling the emotional strain of job loss; most have considered changing occupations. Pew Research Center. Retrieved July 30, 2021 from https://www.pewresearch.org/fact-tank/2021/02/10/ unemployed-americans-are-feeling-the-emotional-strain-of-job-loss-most-haveconsidered-changing-occupations/

[4] Sullivan, P. (2020). CDC director: US has about 28,000 contact tracers, needs 100,000. The Hill, June 23, 2020. Retrieved July 30 from https://thehill.com/policy/healthcare/ 504098-cdc-director-us-has-about-28000-contact-tracers-out-of-100000-needed

[5] Taylor, D.H. (2021). The L\&D global sentiment survey 2021: The impact of COVID. Retrieved July 30, 2021 from https://donaldhtaylor.co.uk/insight/the-ld-global-sentimentsurvey-2021-first-thoughts/

[6] Van Dam, A. (2021). The seven industries most desperate for workers. Washington Post, June 15, 2021.

[7] XPRIZE Foundation (2021). Work reimagined. Retrieved July 30, 2021 from https://www. xprize.org/prizes/rapidreskilling 


\section{Author}

Dr. W. Lewis Johnson is a specialist in artificial intelligence in education. For his work on the first Alelo immersive game, Tactical Iraqi, he won DARPA's Significant Technical Achievement Award. He has been a past President of the International AI in Education Society and was co-winner of the 2017 Autonomous Agents Influential Paper Award for his work in the field of pedagogical agents. Dr. Johnson is President of Alelo, a producer of AI-driven learning products for rapid training and reskilling; 3937 Marcasel Ave., Los Angeles, CA 90066 USA (ljohnson@alelo.com).

Article submitted 2021-08-02. Resubmitted 2021-10-29. Final acceptance 2021-11-02. Final version published as submitted by the authors. 